\title{
RECICLAGEM DE LIXO ELETRÔNICO
}

\section{Dérick da Costa Ferreira}

Grupo de Pesquisa de Eletrônica, telecomunicação e automação, IFRN, Santa Cruz, Bolsista do Programa de Iniciação Científica, Aluno do curso técnico em informática - IFRN. E-mail: phenixdk@hotmail.com.

\section{Josivan Bezerra da Silva}

Grupo de Pesquisa de Eletrônica, telecomunicação e automação, IFRN, Santa Cruz, Bolsista do Programa de Iniciação Científica, Aluno do curso técnico em informática - IFRN. E-mail: jbds1992@yahoo.com.br

\section{Jean Carlos da Silva Galdino}

Grupo de Pesquisa de Eletrônica, telecomunicação e automação, IFRN, Santa Cruz, Orientador, Tec. Eletrônica - ETR; Graduação Eng. Elétrica e Matemática - UFRN; Esp. Em Educação - FACEX; Esp. Em Automação - UNP; Mestrando em Eng. Petróleo - UFRN.

E-mail: Jean.galdino@ifrn.edu.br

\section{RESUMO}

Devido ao grande crescimento da produção de eletro-eletrônicos e a diminuição do tempo de vida dos mesmos aumentamos e muito o e-lixo (lixo eletro-eletrônico) virando um problema. Este artigo apresenta o trabalho de pesquisa em andamento no Instituto Federal de Educação Ciência e Tecnologia do Rio Grande do Norte, voltado para a reciclagem do lixo eletroeletrônicos. Fundamentada em artigos e reportagens da internet e outros meios estão sendo coletados dados sobre o problema do e-lixo na região do trairi do estado do rio grande do norte e no mundo, junto com as informações esta sendo recolhido e-lixo, este é selecionado descaracterizado e classificado em material bom para reuso e os que serão reciclados por empresas industrializadas. Este trabalho encontra-se em andamento, mas já é possível constatar que boa parte do e-lixo pode ser aproveitado seja na mesma linha de produção ou como matéria prima.

PALAVRAS CHAVES: e-lixo, reciclagem, eletrônico.

\section{RECYCLING OF TRASH ELECTRONIC}

\begin{abstract}
Due to the great growth of the production of the electronics and the lifetime decreasing of these same products it has increased and a lot the electronic trash turning itself into a problem. This article presents the work of the ongoing research on the Federal institute of education and science of the state of Rio Grande do Norte which involves the recycling of the electronic trash. Based in articles and interviews from the internet and from other medias they are being collected data about the problem of the electronic trash on the Trairi area and also around the world. Together the information and the electronic trash are being recollected. This is selected uncharacterized and setted as a good material for reuse and the ones which will be recycled by industrialized companies. This job finds itself in a run but it is already possible to
\end{abstract}


ensure that a good part of this trash can be used on the same production line or as a raw material.

KEY-WORD: trash, Recycling, electronic 


\section{RECICLAGEM DE LIXO ELETRÔNICO}

\section{INTRODUÇÃO}

O e-lixo (lixo eletro-eletrônico) é hoje um grande problema mundial, milhões de toneladas de e-lixo são produzidos por ano mundialmente. O problema de tanto e-lixo é que se necessita de uma demanda maior de matéria prima, pra se ter uma idéia a construção de um computador utiliza-se Duas toneladas de matéria prima. Com o crescimento das vendas de eletroeletrônicos e a rápida evolução tecnológica temos dois problemas:

Primeiro um problema ambiental, pois, os eletrônicos são constituídos de metais pesados que estejam sendo descartados no meio ambiente.

Segundo, falta de matéria prima, com a produção de eletrônicos em alta necessita-se de mais matéria prima.

Esse problema existe em diversos países e já está sendo bastante repercutido em todo o mundo, fazendo com que os países dêem mais destaque a esse assunto, sendo criados e desenvolvidos projetos para obter uma solução para esse problema.

Os resíduos eletrônicos já representam $5 \%$ de todo o lixo produzido pela humanidade. $\mathrm{O}$ Brasil produz $2,6 \mathrm{Kg}$ de lixo eletrônico por habitante, o equivalente a menos de $1 \%$ da produção mundial de resíduos do mundo, porém, a indústria eletrônica continua em expansão. Até 2012 espera-se que o número de computadores existentes no país dobre e chegue a 100 milhões de unidades. Baseado na vida útil dos eletro-eletrônicos no prazo de três a cinco anos tudo isso se transformará em lixo tecnológico. Entrarão no mercado anualmente mais 80 milhões de celulares, mas somente $2 \%$ serão descartados de forma correta. Os outros $98 \%$ serão simplesmente guardados em casa ou despejados no lixo comum, criando ainda mais impacto ambiental.

Devido a grande produção de eletrônicos houve um aumento na demanda dos materiais usados na fabricação dos seus componentes, principalmente, dos metais pesados acarretando na diminuição da oferta destes e no aumento de seus preços. De acordo com o professor Ruediger Kuehr da Universidade das Nações Unidas baseado em sua pesquisa diz:

\footnotetext{
Os grandes picos de preços de todos esses elementos especiais que dependem da produção de metais como zinco, cobrem, chumbo ou platina, ressaltam que a manutenção da oferta a preços competitivos não poderá ser garantida indefinidamente a menos que sejam estabelecidos ciclos eficientes de reciclagem para recuperá-los a partir dos produtos obsoletos.(Ruediger Kuehr, 2008).
}

\section{RECICLAGEM}

Reciclar é a alternativa mais viável hoje para o e-lixo. A reciclagem consiste em separar os materiais que compõem um objeto e prepará-los para serem usados novamente como matériaprima dentro do processo industrial. Nem sempre a reciclagem se destina à reinserção dentro do mesmo ciclo produtivo: um computador reciclado pode gerar materiais que vão ser utilizados em outras indústrias. E o material que não da para ser aproveitados é mandado para locais que se desfazem do equipamento que não pode ser utilizado, sem que cause danos ao meio ambiente, evitando problemas futuros como câncer e outras doenças. 
Eis um paradigma econômico-industrial: $\mathrm{O}$ processo conhecido como manufatura reversa de eletrônicos não se paga com a venda dos produtos da reciclagem (sais e óxidos de metais e uma infinidade de plásticos e polímeros), ele só é viável quando é contratado o serviço de reciclar e/ ou neutralizar substâncias tóxicas juntamente com a distinção adequada de resíduos, alem do alto custo esse processo também exige uma tecnologia muito avançada, impedindo que países mais pobres se utilizem de tal recurso. No Brasil as empresas de reciclagem deste lixo apenas o descaracterizam e mandam pra o exterior.

Uma maneira encontrada por países ricos de se livrar do e-lixo é a falsa doação, realizando doações do e-lixo para países pobres com a desculpa de implantar a inclusão digital, sabendo que esses produtos têm pouco ou nenhum tempo de vida.

\section{REMANUFATURAMENTO}

Uma maneira mais econômica de reciclagem seria o remanufaturamento dos eletroeletrônicos. Nesse processo é tentado colocar em funcionamento os eletro-eletrônicos seja na função original ou em outra.esse processo é chamado de reuso - o que pode ser até mesmo um atalho para a inclusão digital. O governo federal, por exemplo, conta com quatro centros de recondicionamento de computadores (em Porto Alegre, Guarulhos, Brasília e Belo Horizonte) que dão uma segunda vida aos equipamentos. Depois de reparados e atualizados, são doados a escolas públicas, bibliotecas, entre outras instituições. Mais dois centros - na Bahia e no Pará - estão em fase de implantação.

Caso o aparelho não seja colocado em funcionamento o mesmo é descaracterizado, os componentes bons são aproveitados para outros fins e o restante é mandado para a reciclagem especifica para cada tipo de material.

Ainda existem pouquíssimas empresas de reciclagem do e-lixo no Brasil, desses, a maioria apenas recebe o e-lixo, descaracteriza e repassa para empresas especializadas, geralmente fora do País.

\section{FUNDAMENTAÇÃO TEÓRICA}

Segundo site ecoamigos.wordpress.com, considera-se lixo tecnológico (ou e-lixo) todo aquele gerado a partir de aparelhos eletrodomésticos ou eletroeletrônicos e seus componentes, incluindo os acumuladores de energia (baterias e pilhas) e produtos magnetizados, de uso doméstico, industrial, comercial e de serviços, que estejam em desuso e sujeitos à disposição final. O mundo joga fora cerca de 50 milhões de toneladas de sucata eletrônica por ano, entre computadores, celulares, televisores e aparelhos de som.

Em 2007, os brasileiros compraram 20 milhões de computadores, 11 milhões de televisores e 21 milhões de novos telefones celulares. A legislação ambiental brasileira trata os resíduos pelo elemento contaminante e determina o seu tratamento, porém, apenas alguns manufaturados dispõem de normas legais de descarte, como as PILHAS e BATERIAS.

Segundo Paulo Roberto leite e natale lavez et al. (2009), Afirmam que em um estudo coordenado pelo professor Ruediger Kuehr da Universidade das Nações Unidas, os pesquisadores descobriram que nada menos de 1,8 toneladas de materiais dos mais diversos tipos são utilizados para se construir um único computador. Além do desperdício e do seu 
grande potencial poluidor e até mesmo tóxico, o chamado e-lixo, ou lixo eletrônico, está fazendo um estrago nas cotações dos metais utilizados na fabricação de componentes e circuitos eletrônicos.

"Os produtos são fabricados usando mais de mil químicos tóxicos muitos deles são conhecidos por causar câncer, abortos, problemas reprodutivos, asma e outras doenças nos trabalhadores que os fabricam, nas comunidades que cercam as fábricas e em locais onde o elixo é jogado e queimado", afirma Ted Smith, autor do livro "Challenging the Chip" (desafiando o chip).

Segundo o CEMPRE: Compromisso Empresarial para Reciclagem, os resíduos eletrônicos já representam 5\% de todo o lixo produzido pela humanidade. O Brasil produz 2,6Kg de lixo eletrônico por habitante, o equivalente a menos de $1 \%$ da produção mundial de resíduos do mundo, porém, a indústria eletrônica continua em expansão. Até 2012 espera-se que o número de computadores existentes no país dobre e chegue a 100 milhões de unidades. Entrarão no mercado anualmente mais 80 milhões de celulares, mas somente $2 \%$ serão descartados de forma correta. Os outros $98 \%$ serão simplesmente guardados em casa ou despejados no lixo comum, criando ainda mais impacto ambiental.

Segundo site licenciamentoambiental.eng.br, "No prazo de três a cinco anos tudo isso se transformará em lixo tecnológico", afirma Rodrigo Baggio, diretor executivo do Comitê para Democratização da Informática (CDI). De acordo com os dados da Secretaria do Meio Ambiente do Estado de São Paulo, o tempo médio de degradação dos metais, que inclui componentes de equipamentos, é de quase meio milênio. Preocupado com o crescimento desse lixo e com a falta de regulamentação e debates que conscientizem a população brasileira sobre os sérios riscos provocados, Baggio enxerga um futuro nada promissor ao meio ambiente e à saúde humana brasileira. "O Brasil ainda não tem um plano de reciclagem e de descarte seguro para o lixo tecnológico", reforça. "Nos Estados Unidos e Europa, ao contrário do Brasil, existem empresas dedicadas à reciclagem tecnológica e reaproveitamento dos produtos, neutralizando os componentes tóxicos", conta Rodrigo Baggio, diretor executivo do Comitê para Democratização da Informática (CDI).

Segundo o site ambientebrasil.com.br, a Constituição Federal Brasileira, promulgada em 1988, trata de forma abrangente e moderna os assuntos relacionados à preservação do meioambiente e ao desenvolvimento sustentável da economia, reservando a união, aos estados, ao distrito federal e aos municípios, a tarefa de proteger o meio ambiente e de controlar a poluição (artigo 23). O gerenciamento dos resíduos sólidos de origem domiciliar, que inclui o grupo dos e-lixo, apresenta tratamento diferenciado em diferentes partes do país. Enquanto alguns municípios têm por prática intervir e monitorar amplamente o descarte dos produtos, outros ainda não dispõem de uma abordagem adequada para o tratamento e-lixo.

Segundo site lixoeletronico.org, reciclar é a alternativa mais viável hoje para o e-lixo.A reciclagem consiste em separar os materiais que compõem um objeto e prepará-los para serem usados novamente como matéria-prima dentro do processo industrial. Nem sempre a reciclagem se destina à reinserção dentro do mesmo ciclo produtivo: um computador reciclado pode gerar materiais que vão ser utilizados em outras indústrias mas existem pouquíssimas empresas no Brasil que realizam todo o processo de separação e preparação de materiais para a reciclagem. Eis um paradigma econômico-industrial: $\mathrm{O}$ processo conhecido como manufatura reversa de eletrônicos não se paga com a venda dos produtos da reciclagem (sais e óxidos de metais e uma infinidade de plásticos e polímeros), ele só é viável quando é 
contratado o serviço de reciclar e ou neutralizar substâncias tóxicas juntamente com a distinção adequada de resíduos.

Segundo site amanha.com.br, outra solução para o e-lixo é o reuso - o que pode ser até mesmo um atalho para a inclusão digital. O governo federal, por exemplo, conta com quatro centros de recondicionamento de computadores (em Porto Alegre, Guarulhos, Brasília e Belo Horizonte) que dão uma segunda vida aos equipamentos. Depois de reparados e atualizados, são doados a escolas públicas, bibliotecas, entre outras instituições. Mais dois centros - na Bahia e no Pará - estão em fase de implantação.

\section{OBJETIVO}

\section{GERAL:}

Essa pesquisa tem como objetivo obter informações de como o e-lixo esta sendo descartado no Brasil com foco na região do trairi do estado do rio grande do norte, abordando aspectos como os problemas gerados e as possíveis soluções e alternativas, como e onde reciclar este lixo e através de experiências sem processos químicos descobrir novas formas que sejam economicamente viáveis e ecológicas de reciclar o e-lixo (ou lixo eletrônico). Com os resultados da pesquisa conscientizar a população da região trairi do problema que é hoje o lixo eletrônico e os orientar de como descarta ou reutilizar o seu e-lixo.

\section{ESPECÍFICOS:}

Coletar de dados

Estabelecer postos de coleta e recolher e-lixo

Descaracterizar o material recolhido

Realizar experiências com o e-lixo coletado

Com os resultados da pesquisa conscientizar a população através de palestras

\section{METODOLOGIA}

Coletando dados sobre como esta sendo descartado o lixo eletrônico no Brasil e onde estão localizadas as empresas que reciclam o e- lixo é feito uma estimativa do tipo de material que é mais abundante neste tipo de lixo, que irar servir de base para a pesquisa. $\mathrm{O}$ material para estudo esta sendo obtida com divulgação da pesquisa em meios de comunicação possíveis e estabelecendo postos de coleta de lixo eletrônico. Após acumular material de pesquisa, o mesmo estar sendo separado em aqueles que serão colocados em funcionamento e os que serão descaracterizados. Os materiais descaracterizados serão testados e separados em funcionais e não funcionais, os funcionais serão utilizados em outros projetos e os não funcionais irão para as empresas de reciclagem para voltarem a ser matéria prima. por fim usar os dados obtidos para conscientizar a população através de palestras de como se deve descartar seu lixo eletrônico sem agredir o meio ambiente. 


\section{RESULTADOS OBTIDOS}

Com a pesquisa constatou-se que pelo menos $80 \%$ do e-lixo pode ser reaproveitado (Gráfico 1), isto baseado nos materiais obtidos, testados e reutilizados nesta pesquisa e no fato de que os eletro-eletrônicos cada vez mais têm seu tempo de vida reduzido devido o rápido avanço tecnológico sendo descartados cada vez mais peças em bom estado e funcionamento. Com material recolhido e descaracterizado foi obtidos componentes em bom estado que estão sendo usados no IFRN em reparos de outros eletro-eletrônicos que voltaram a serem utilizados, em outros projetos acadêmicos de eletrônica e utilizados pelos professores para dar aulas em cursos tecnológicos (FIGURA 1), os materiais não aproveitados serão separados e mandados para sua respectiva área de reciclagem.

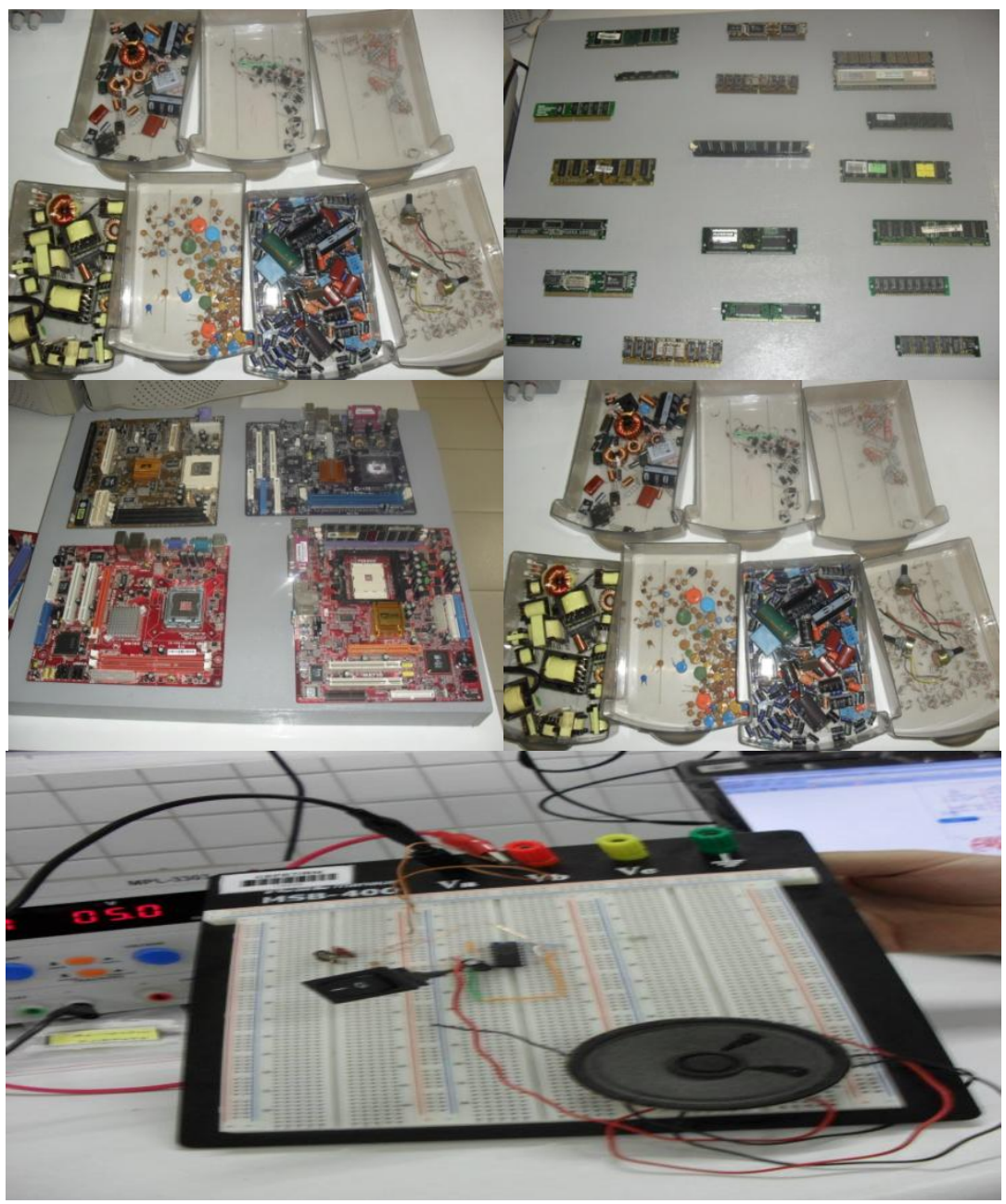

Figura 1 - Lixo reciclado na pesquisa. 


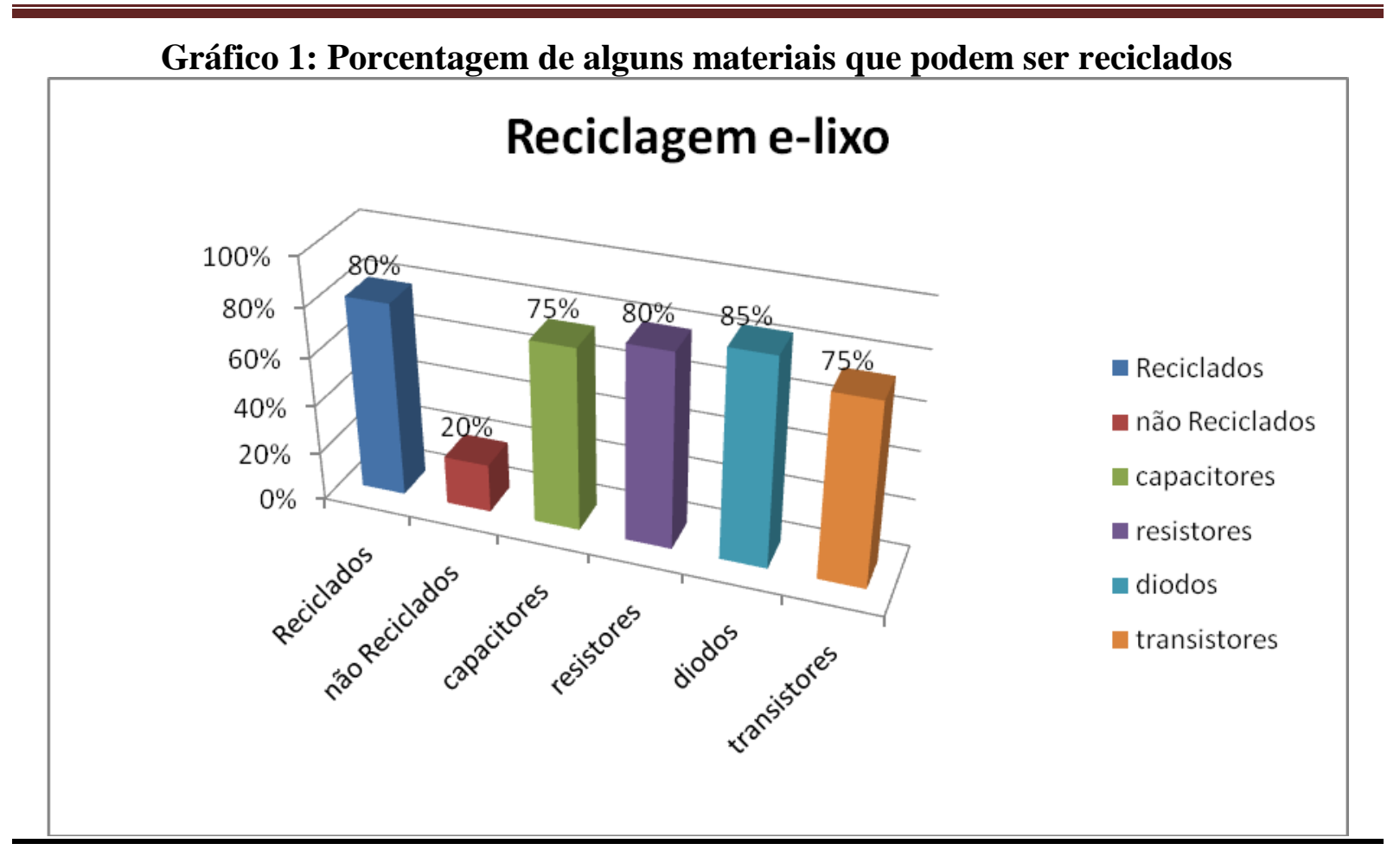

\section{RESULTADOS ESPERADOS}

Com esta pesquisa espera-se obter meios eficazes de reutilizar o lixo eletrônico, saber como e onde este lixo esta sendo descartado e reciclado no Brasil com foco na região do trairi do estado do rio grande do norte e conscientizar a população local do problema do lixo eletrônico.

\section{REFERÊNCIAS BIBLIOGRÁFICAS}

1. IDOETA, IVAN VALEIJE. Elementos de Eletrônica Digital. São Paulo: Érica, 2007.

2. MALVINO, ALBERT PAUL. Eletrônica: Vol. 1. São Paulo: PEARSON, 2010.

3. MILlMAN, J., HALKIAS, C. C. Eletrônica: dispositivos e circuitos. São Paulo: Editora McGraw-Hill do Brasil, 1981.

4. SEDRA, Adel S. Kenneth C. Smith. Microeletrônica. São Paulo: Pearson Prentice Hall, 2007;

5. VAHID, Frank. Sistemas digitais: Projetos, otimização e HDLS. Porto Alegre: Artmed, 2008.

6. NEBOJSA, Matic. Microcontroladores PIC. Ed. Érica, São Paulo, 2008;

7. CEMPRE: Compromisso Empresarial para Reciclagem. Apresenta informações sobre reciclagem. Disponível em: <http://www.cempre.org.br>. Acesso em: 25.set.2007.

8. LEITE, PAULO ROBERTO et al. Fatores Da Logística Reversa Que Influem No Reaproveitamento Do "Lixo Eletrônico" - Um Estudo No Setor De Informática. SIMPO I, 2009.

9. Rosa, Agostinho (2007). Fabricação de cada computador consome 1.800 quilos de materiais. 
http://www.inovacaotecnologica.com.br/noticias/noticia.php?artigo=010125070309.

10. Fonseca, Felipe (2008). O ciclo do Lixo Eletrônico - 1. Produção e consumo. Acedido em: 30.09.2008, em: http://lixoeletronico.org/blog/o-ciclo-do-lixo-eletr\%C3\%B4nico-1produ\%C3\%A7\%C3\%A3o-e-consumo.

11. Fonseca, Felipe (2008). O ciclo do Lixo Eletrônico - 2. Descarte e reuso. Acedido em: 02.10.2008, em: http://lixoeletronico.org/blog/o-ciclo-do-lixo-eletr\%C3\%B4nico-2descarte-e-reuso.

12. Fonseca, Felipe (2008). O ciclo do Lixo Eletrônico - 3. Reciclagem. Acedido em: 04.11.2008, em: http://lixoeletronico.org/blog/o-ciclo-do-lixo-eletr\%C3\%B4nico-3reciclagem.

13. Andueza, Felipe (2008). O paradigma econômico da reciclagem do lixo eletrônico. Acedido em: 26.11.2008, em: http://lixoeletronico.org/blog/o-paradigmaecon\%C3\%B4mico-da-reciclagem-do-lixo-eletr\%C3\%B4nico.

14. Viana, José (2008). Lixo tecnológico . Acedido em: 20/10/2008, em: http://ecoamigos.wordpress.com/2008/10/20/lixo-tecnologico/.

15. Coelho, Luiza (2008). Lixo tecnológico deve triplicar nos próximos 5 anos. Acedido em: 06/06/2008, em: http://www.licenciamentoambiental.eng.br/lixo-tecnologico-devetriplicar-nos-proximos-5-anos/.

16. Smaal, Beatriz Portella (2009). Lixo eletrônico: o que fazer após o término da vida útil dos seus aparelhos?. Acedido em: 11 /08/2009, em: http://www.baixaki.com.br/info/2570-lixo-eletronico-o-que-fazer-apos-o-termino-davida-util-dos-seus-aparelhos-.htm.

17. Forum Revista Amanhã (2008). Lixo Tecnológico. Acedido em: 15/12/2009, em: http://www.amanha.com.br/NoticiaDetalhe.aspx?NoticiaID=943088a0-1f6d-46d28b54-0efbb206fa3a.

18. Portal Brasil Ambiental (2009). Tratamento de lixo tecnológico - no Brasil e na União Européia. Acedido em: 25/10/2010, em: http://www.ambientebrasil.com.br/composer.php3?base=residuos/index.php3\&conteud $\mathrm{o}=. /$ residuos/artigos/trat_lixo.html.

19. Blog do Instituto Sergio Motta. (2009). Lixo Eletronico no Brasil. Acedido em: 06/02/2009, em: http://blog.premiosergiomotta.org.br/2009/02/04/lixo-eletronico-nobrasil/. 